\title{
Hydrolytically Labile Linkers Regulate Release and Activity of Human Bone Morphogenetic Protein-6
}

\author{
Jordi Cabanas-Danés, ${ }^{\dagger,}$ Ellie Landman, ${ }^{\S}$ Jurriaan Huskens, ${ }^{\ddagger \odot}$ Marcel Karperien, ${ }^{*}, \S$ \\ and Pascal Jonkheijm $*, t, \ddagger$
}

\begin{abstract}
${ }^{\dagger}$ Bioinspired Molecular Engineering Laboratory, TechMed Centre, University of Twente, P.O. Box 217, 7500 AE Enschede, The Netherlands

${ }^{\ddagger}$ Molecular Nanofabrication Group, MESA+ Institute for Nanotechnology, University of Twente, P.O. Box 217, 7500 AE Enschede, The Netherlands

${ }^{\S}$ Developmental BioEngineering Group, TechMed Centre, University of Twente, P.O. Box 217, 7500 AE Enschede, The Netherlands
\end{abstract}

\section{Supporting Information}

ABSTRACT: Release of growth factors while simultaneously maintaining their full biological activity over a period of days to weeks is an important issue in controlled drug delivery and in tissue engineering. In addition, the selected strategy to immobilize growth factors largely determines their biological activity. Silica surfaces derivatized with glycidyloxy propyl trimethoxysilane and poly(glycidyl methacrylate) brushes yielded epoxide-functionalized surfaces onto which human bone morphogenetic protein-6 (hBMP-6) was immobilized giving stable secondary amine bonds. The biological activity of hBMP- 6 was unleashed by hydrolysis of the surface siloxane and ester bonds. We demonstrate that this type of labile bonding strategy can be applied to biomaterial surfaces with relatively simple and biocompatible chemistry, such as siloxane, ester, and imine bonds. Our data indicates that the use of differential hydrolytically labile linkers is a versatile method for functionalization of biomaterials with a variety of growth factors providing control over their biological activity.

\section{INTRODUCTION}

Growth factors (GFs) are endogenously present either bound to the extracellular matrix $(\mathrm{ECM})^{1}$ or as soluble molecules secreted by cells. Bound GFs, as opposed to their soluble analogues, provide control over their local concentration and protection from enzymatic degradation. ${ }^{2}$ Many growth factors are bound in an inactive conformation to the ECM. Upon proteolytic cleavage, these growth factors become active and are released in the extracellular space. An example of such a GF is the Bone Morphogenetic Protein-6 (hBMP-6) growth factor, which is a member of the transforming growth factor- $\beta$ (TGF$\beta$ ) superfamily. hBMP-6 is stored in the ECM as an inactive precursor molecule. Upon proteolytic cleavage, active, mature hBMP- 6 is released from the degrading ECM which in turn stimulates cells to repair the lost tissue. ${ }^{3}$ BMP- 6 is known to regulate differentiation of various cells implicated in cartilage and bone formation during skeletal development and fracture repair. $^{4-6}$ This is exemplified by the hBMP-6 mediated induction of alkaline phosphatase activity in osteoblast precursor cells, a hallmark of osteogenic differentiation. ${ }^{7}$

Throughout the past decade, many efforts have been devoted to develop methods to immobilize GFs at biomaterial surfaces. $^{8-10}$ The main aim of these efforts is to mimic the endogenous GF delivery system and thereby improving the performance of biomaterials in tissue engineering and therapeutic applications. ${ }^{8-10}$ For example, hBMP-2 has been mixed with biomaterials to be released by diffusion and thereby activating cell differentiation. ${ }^{11,12}$ Other approaches have been focusing on linking ECM elements, such as heparins, ${ }^{13-15}$ or mimics thereof, ${ }^{16-20}$ to biomaterials in order to position growth factors on their surfaces through heparin's growth factor binding domains. Single-domain antibodies have also been employed to reversibly link growth factors to surfaces, ${ }^{21}$ or alternatively, direct coupling of growth factors has also been pursued using noncovalent His-tag technology. ${ }^{22}$ Covalent chemistry yields long-term presentation of growth factors, ${ }^{23,24}$ which continuously engage in receptor binding and protein dimerization, and hamper internalization of growth factors. ${ }^{25}$

Here, we investigate the effect on cells when hydrolytically labile siloxane and imine bonds are used as a linkage between the surface and the GF. Such chemistry has been successfully applied for the controlled formation of for example reversible gels, polymers and functional surfaces. ${ }^{26-28}$ Siloxane and imine bonds are attractive as they can be hydrolyzed under physiological conditions. ${ }^{29-34} \mathrm{Klok}$ and co-workers have studied the stability of trialkoxysilane-anchored brushes and

Received: March 15, 2018

Revised: May 24, 2018

Published: July 13, 2018 


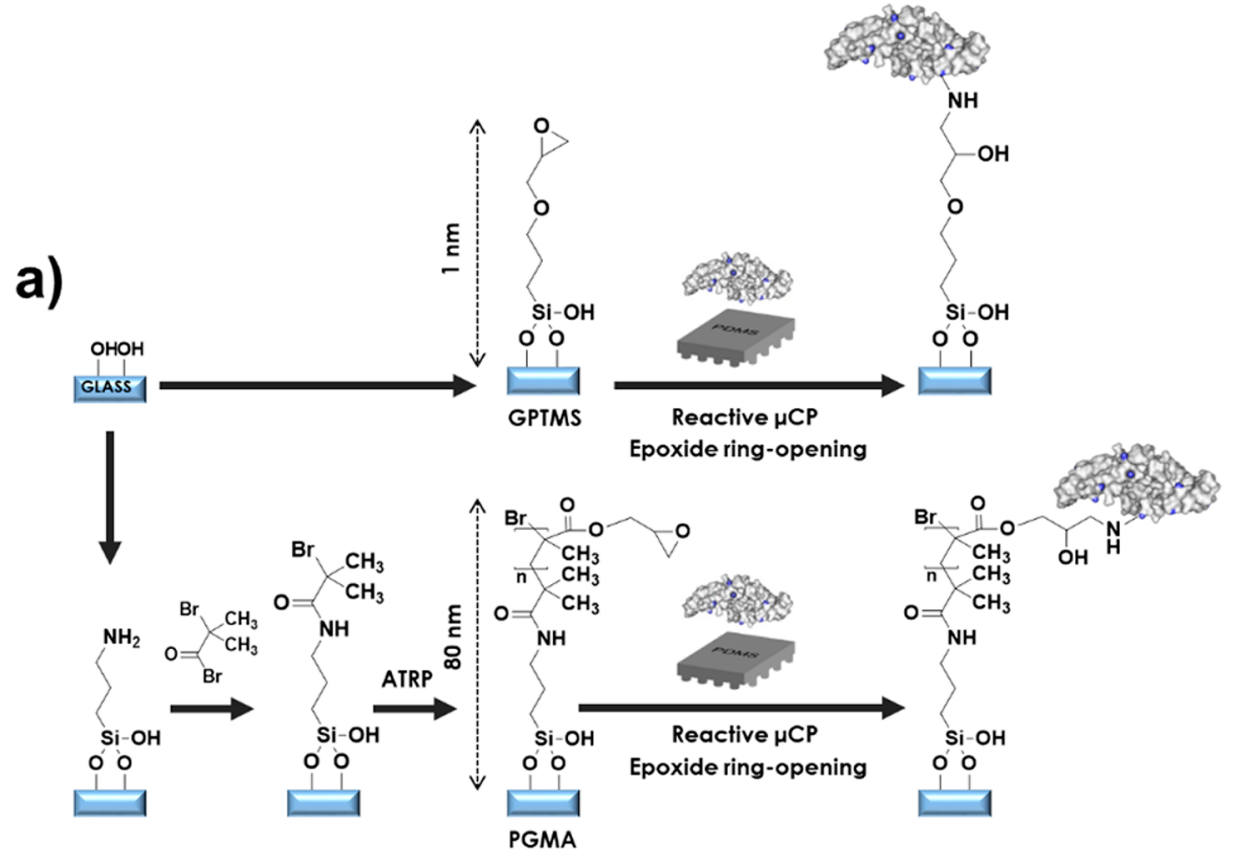

b)

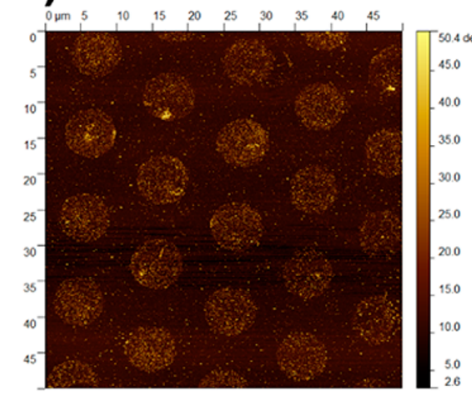

c)

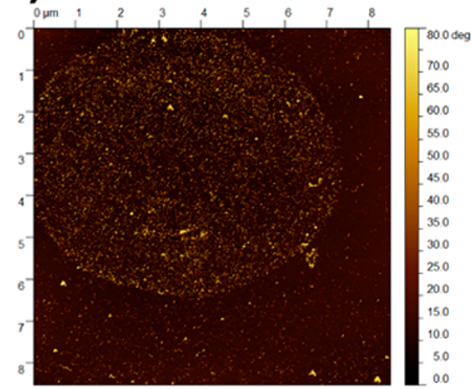

d)

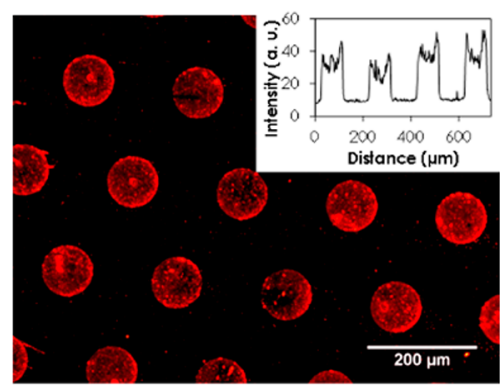

Figure 1. (a) Schematic overview of the preparation of epoxide-terminated glass substrates with two linkers of different length that have a difference in hydrolytic release: (3-glycidyloxypropyl)trimethoxysilane (GPTMS) (top) and poly(glycidyl methacrylate) (PGMA) (bottom) and subsequent immobilization of hBMP-6 (accessible lysine residues are indicated in dark gray) by reactive microcontact printing ( $\mu \mathrm{CP}$ ) (atom transfer radical polymerization (ATRP)). (b) Atomic force microscopy (AFM) phase image of hBMP-6 micropatterned (10 $\mu$ m diameter dots and $5 \mu \mathrm{m}$ spacing) on an epoxide-terminated surface without using a passivated background. (c) Zoom in of the phase image. (d) Fluorescence microscopy image (inset shows the intensity profile) of immunostained $\mu \mathrm{CP}$ patterns of hBMP-6 (100 $\mu \mathrm{m}$ diameter dots and $100 \mu \mathrm{m}$ spacing) on an epoxide-terminated surface using a background passivated by $\mathrm{NH}_{2}$-PEG (see the Supporting Information for details).

found that brushes detached from the surface upon prolonged exposure to cell culture medium. ${ }^{3 \mathrm{~b}}$ Using reversible imine bonds, biologically active molecules were cross-linked in a hydrophilic polymer network. ${ }^{29}$ Differences in the percentage of released small molecules as well as the duration of that release were strictly depended on the percentage of the entrapped compound in the solvent of the gel. ${ }^{29}$ In addition, reversible imine bonds were used to fabricate various mixed surface patterns and gradients to control surface compositions of small molecules and their subsequent release. ${ }^{31,32}$ Despite the potential of adopting reversible chemistry for constructing and functionalizing materials and surfaces, to date these types of reversible siloxane and imine bonds have not been explored for the delivery of GFs. We study the effect on cell response of releasing hBMP-6 through hydrolytical cleavage. Release can be slowed down by creating a polymer brush. The data suggests the released growth factors are bioactive.

\section{RESULTS AND DISUCSSION}

The Immobilization of hBMP-6 onto Epoxide-Coated Surfaces and Its Cell Signaling Capability. Monolayers of hBMP-6. To establish the method of hydrolytically releasing hBMP-6, we started with model studies on freshly prepared epoxide-terminated glass slides. Deposition of (3glycidyloxypropyl)trimethoxysilane (GPTMS, Figure 1a) successfully yielded monolayers, evident from the recorded advancing contact angle value of $55^{\circ} \pm 2^{\circ}$ and the increase in film thickness of $0.9 \mathrm{~nm} \pm 0.2 \mathrm{~nm}$ using ellipsometry, which is in agreement with literature (Table 1). ${ }^{33,34}$ hBMP-6 was covalently immobilized to epoxide-functionalized substrates by reactive microcontact printing $(\mu \mathrm{CP})^{35}$ (Figure 1a) through a nucleophilic ring-opening reaction, presumably with primary amine groups of the lysine residues that are localized at the periphery of the dimeric GF structure. Immobilization of hBMP-6 led to an increase of the advancing contact angle value from $55^{\circ} \pm 2^{\circ}$ to $64^{\circ} \pm 5^{\circ}$, to the appearance of an N1s peak in the XPS spectrum indicating the presence of ca. $1 \% \mathrm{~N}$ and to a further increase in film thickness of $1 \mathrm{~nm}$ as observed by 
Table 1. Advancing $\left(\boldsymbol{\theta}_{\mathrm{a}}\right)$ and Receding $\left(\boldsymbol{\theta}_{\mathrm{b}}\right)$ Water Contact Angle Values, Ellipsometric Thicknesses, and $N$ Fractions Determined by XPS of the Two Epoxide-Terminated Linkers before and after the Immobilization of hBMP-6

\begin{tabular}{lcccc} 
monolayer (full) & $\theta_{\mathrm{a}}[\mathrm{deg}]$ & $\theta_{\mathrm{b}}[\mathrm{deg}]$ & $\begin{array}{c}\text { ellipsometric } \\
\text { thickness [nm] }\end{array}$ & $\begin{array}{c}N[\%] \\
\text { (XPS) }\end{array}$ \\
GPTMS & $55 \pm 2$ & $40 \pm 5$ & $0.9 \pm 0.2$ & $-^{a}$ \\
GPTMS + hBMP-6 & $64 \pm 5$ & $47 \pm 2$ & $1.9 \pm 0.2$ & $1.0 \pm 0.3$ \\
PGMA & $60 \pm 1$ & $52 \pm 4$ & $80 \pm 3$ & $-^{a}$ \\
PGMA + hBMP-6 & $65 \pm 1$ & $41 \pm 4$ & $87 \pm 2$ & $0.7 \pm 0.2$ \\
${ }^{a}$ Not detected. & & & & \\
\hline
\end{tabular}

ellipsometry (Table 1). Inspection of the monolayers using atomic force microscopy (AFM) (Figure 1b, c) revealed clear patterns of effectively immobilized hBMP-6 with on average nearly $70 \%$ of the total area of the pattern occupied with protein. A height of $0.5 \mathrm{~nm}$ between the protein patterns and the background was observed (Figure S1), which is lower than observed using ellipsometry presumably due to tip-induced compression of the proteins. These results indicate that hBMP6 is immobilized in a moderately packed, single layer configuration, which amounts to ca. $120 \mathrm{ng} / \mathrm{cm}^{2}$.

Induction of Osteogenic Differentiation. Immobilized hBMP-6 was also visualized using immunofluorescence staining of the surface. The recorded fluorescence micrograph (Figure 1d) showed clearly the presence of hBMP-6 at the surface. This result indicates that the immobilized hBMP-6 had conceivably retained the GF secondary structure, which is essential for antibody binding. The covalent immobilization appeared to be specific to the epoxide SAM because in controls with preblocking the epoxide groups with BSA or incubation on a glass surface, the immunofluorescence staining was absent. To determine whether immobilized hBMP-6 remained biologically active on the surface, a mesenchymal precursor cell line (KS483) was seeded for $24 \mathrm{~h}$ on the surfaces. Cells adhered on glass in the absence of hBMP-6, while addition of
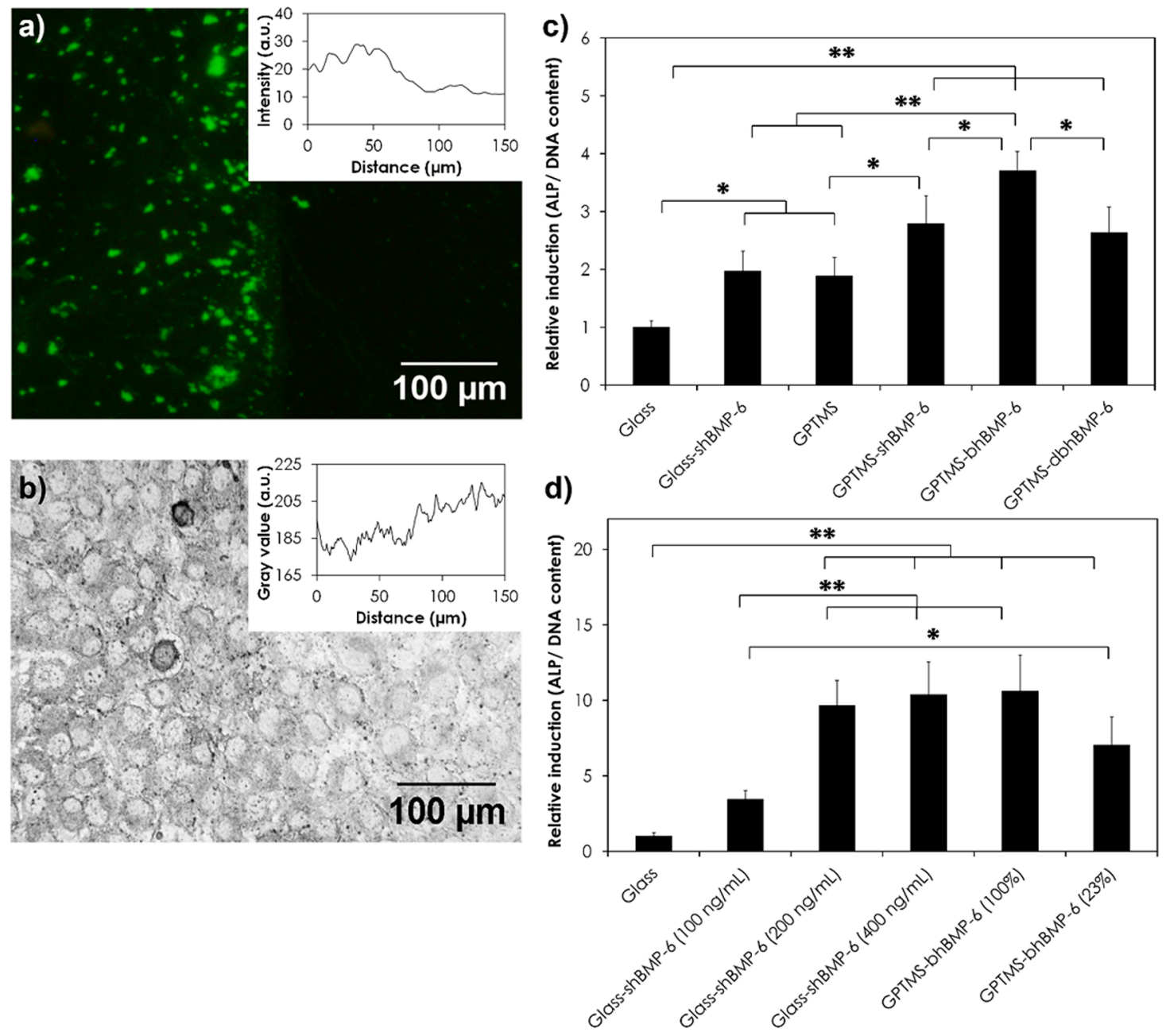

Figure 2. Study of early osteogenesis in KS483 cells by immobilized hBMP-6. (a) Fluorescence microscopy image of hBMP-6 immobilized (by immersion $2 \mathrm{~h}$ in this case) on half of the substrate after immunofluorescence staining (inset shows averaged intensity profiles). (b) Immunohistochemical staining of ALP, after 4 days by KS483 cells (inset shows intensity profile with high (white) intensity indicative of low ALP activity). This slide was also prepared by immobilization of hBMP-6 by immersion $(2 \mathrm{~h}$ ). (c) ALP activity normalized by the total DNA content of KS483 cells after 6 days of culture, expressed as relative induction to a glass control in the presence of soluble $(\mathrm{s}(100 \mathrm{ng} / \mathrm{mL}))$, bound $(\mathrm{b})$, denatured bound (db) hBMP-6. Immobilization of hBMP-6 using flat stamps. (d) Dose response of KS483 cells cultured for 7 days in the presence of increasing concentrations of hBMP-6 in solution and two different amounts of bound hBMP-6, i.e., a full layer $\left(100 \%, \mathrm{ca} .120 \mathrm{ng} / \mathrm{cm}^{2}\right)$ of hBMP6 and a $\mu \mathrm{CP}$ patterned layer of hBMP-6 (23\% of the coverage in comparison to the full layer (using a flat stamp, ca. $\left.28 \mathrm{ng} / \mathrm{cm}^{2}\right) . * p<0.05$ and $* * p$ $<0.01$. 
hBMP-6 or immobilized hBMP-6 on GPTMS layers did not lead to significant changes in cell spreading kinetics, as expected (Figure S2). Actin cytoskeleton stains showed no significant differences in cell area and stress fiber formation (Figure S2). Confluency was reached after 4 days, and no cytotoxicity of the surfaces was observed (Figure S2). KS483 cells can differentiate into osteoblasts during a 1-3 week culture period under osteogenesis-inducing conditions and this can be shown by the induction of alkaline phosphatase (ALP) as early marker of osteogenic differentiation. Mature human and mouse BMP-6 share 96\% amino acid identity, and human BMPs cross react with mouse BMP receptors. This cell model is therefore suitable to assess the ability of hBMP- 6 to initiate differentiation into mineralizing osteoblasts. ${ }^{21,36}$ KS483 cells were seeded on immobilized hBMP- 6 coated surfaces and both an immunofluorescence hBMP-6 stain and an immunohistochemical ALP stain were clearly visible in contrast to control surfaces with immobilized polyethyleneglycol (Figure 2a, b). These results indicate that only in the areas where functional hBMP-6 was available, ALP production was induced. The biological activity of immobilized hBMP- 6 and supplemented hBMP-6 was compared by normalizing the ALP activity in KS483 cells after seven days of culture to the DNA content of the total number of cells in contact with the substrate and expressed as relative induction values compared to a glass control (Figure 2c). Interestingly, cells seeded on immobilized hBMP-6 produced significantly more ALP when compared to all other cases (Figure 2c). Increase in induced ALP activity on immobilized hBMP-6 was 4-fold in comparison with controls on glass. This contrasts significantly with the 2 -fold increase in induced ALP activity when the culture medium was supplemented with comparable amounts of hBMP-6. Controls where hBMP-6 was partly denatured (by heating at $90{ }^{\circ} \mathrm{C}$ for 2 min) prior to cell seeding showed a significant loss of ALP. Controls where cells were seeded on epoxide-terminated glass, in medium supplemented with hBMP-6, showed a significantly lower ALP activity when compared to immobilized hBMP-6. Yet, the ALP induction was significantly higher when compared to epoxide-terminated glass controls without supplemented hBMP-6 and glass controls with supplemented hBMP-6. Also, epoxide-terminated surfaces induced ALP activity by 2 -fold versus glass controls indicating an osteoinductive effect of the epoxide groups. Dose-dependent ALP activities were performed for immobilized and supplemented hBMP-6 (Figure 2d). When supplemented hBMP-6 was doubled to $200 \mathrm{ng} / \mathrm{mL}$, a significant rise in ALP activity was noted, but the ALP activity plateaued when hBMP-6 was doubled again. A concentration of $200-400 \mathrm{ng} / \mathrm{mL}$ hBMP-6 is in excess. ${ }^{37}$ Interestingly, the ALP activity of cells seeded on immobilized hBMP-6 compared favorably to the case when $200 \mathrm{ng} / \mathrm{mL}$ or higher of supplemented hBMP- 6 was used. This indicates that a sufficient amount of active hBMP-6 is available on the surface (ca. $120 \mathrm{ng} / \mathrm{cm}^{2}$ ) to achieve the maximum ALP activity. The immobilized hBMP- 6 dose was easily reduced by a fourth (ca. $28 \mathrm{ng} / \mathrm{cm}^{2}$ ) using a featured $\mu \mathrm{CP}$ stamp during the immobilization step. This reduced dose resulted in a significantly lower ALP activity when compared to an entirely covered surface, but still significantly higher when compared to $100 \mathrm{ng} / \mathrm{mL}$ of hBMP-6 that was supplied to the medium. Taken together, these results indicate that immobilized hBMP6 is bioactive and effective to induce ALP activity in cells.

Shielded Release of hBMP-6. We next attached hBMP-6 to the surface by essentially similar chemistry, but now using a longer brush-type linker. ${ }^{38}$ To this end, layers of poly(glycidyl methacrylate) (PGMA) brushes on glass were prepared (Figure 1a). ${ }^{38-42}$ Briefly, the atom transfer radical polymerization (ATRP) initiator, 2-bromo-2-methylpropionyl bromide, was reacted with the terminal amine moieties of a (3aminopropyl)triethoxysilane (APTES) monolayer (Figure 1a). Subsequently, the immobilized initiator was immersed in an oxygen-free solution of glycidyl methacrylate as the ATRP monomer for $2 \mathrm{~h}$ to yield a PGMA brush layer of $80 \mathrm{~nm}$ thickness (Table 1). Immobilization of hBMP-6 onto the PGMA layers was confirmed using contact angle, ellipsometry and XPS measurements (Table 1). Immunofluorescence stains of hBMP-6 on PGMA brushes were of similar intensity as compared to the hBMP-6 patterns on GPTMS surfaces (Figures 1d and S3) suggesting similar loading of hBMP-6 on either the GPTMS or PGMA coated surfaces. The release of hBMP-6 in culture medium was quantified using an enzymelinked immunosorbent assay (ELISA) over a period of 15 days without cells seeded (Figure 3 top). In the case of the short
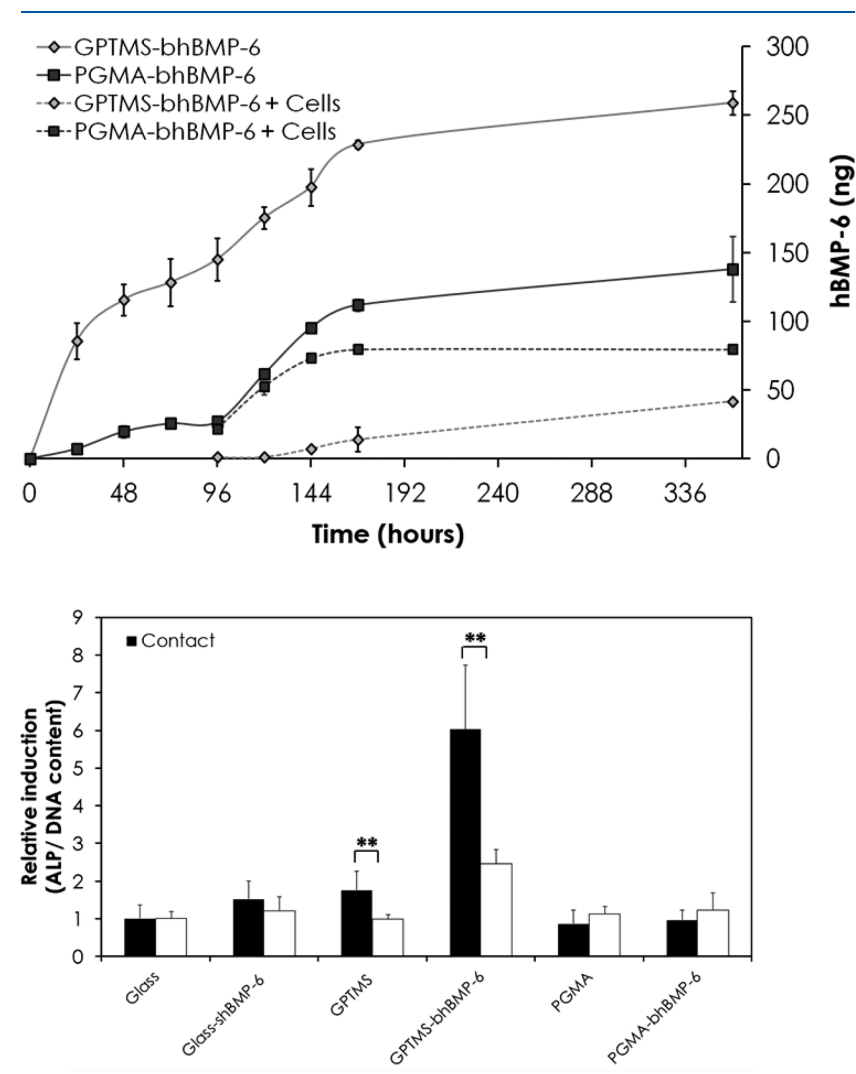

Figure 3. Quantification of hBMP-6 in medium by ELISA (top) over a period of 15 days without (solid lines) and with (dashed lines) 5000 cells $/ \mathrm{cm}^{2}$ on GPTMS and PGMA with initially $\mu \mathrm{CP}$ hBMP-6 (patterned stamps). Relative induction of ALP by KS483 cells after 4 days relative to a glass control (bottom). Black bars represent relative induction of cells seeded in contact with the films. White bars represent relative induction of cells seeded in transwell. $* * p<0.01$.

(GPTMS) linker, a released quantity of $100 \mathrm{ng} / \mathrm{mL}$ hBMP-6 was promptly reached within $24 \mathrm{~h}$, which moderately increased and plateaued in the following 7 days. The concentration of $100 \mathrm{ng} / \mathrm{mL}$ of hBMP-6 is a therapeutic level reported in literature. ${ }^{37,43}$ In the case of the long, PGMA linker, the release of hBMP-6 was much slower (by a factor 6-10) in the first 4 days and the therapeutic hBMP-6 level of $100 \mathrm{ng} / \mathrm{mL}$ was only 
a)
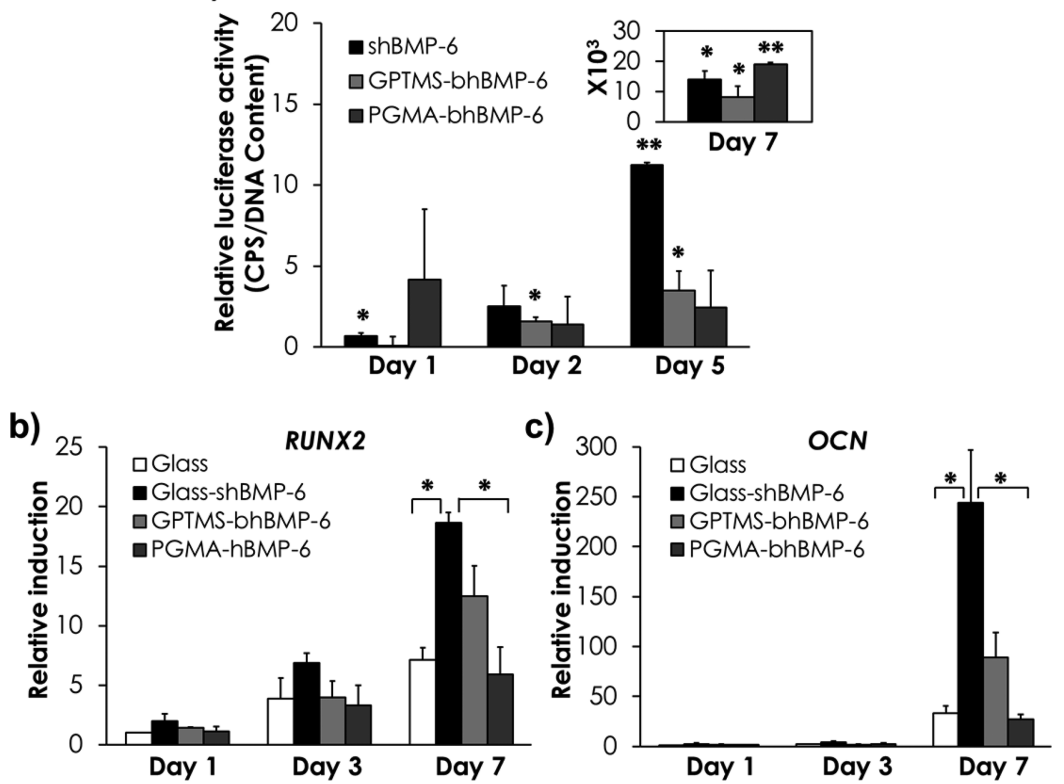

Figure 4. (a) Luminescence of $\mathrm{C} 2 \mathrm{C} 12$ cells transfected with BRE-Luc, measured at different time points relative to a background using the same surface without hBMP-6. Flat stamps were used for immobilization of hBMP-6. Inset shows the luminescence results at day 7 from an independent experiment. Gene expression analysis at days 1, 3, and 7 of (b) RUNX2 and (c) OCN was determined via the $2 \Delta \Delta \mathrm{Ct}$ method using the expression on day 1 on glass as a reference. $* p<0.05$ and $* * p<0.01$.

reached at day 5, while the total amount of released hBMP-6 after 7 and 15 days was half of that on GPTMS. When the ELISA assay was performed when cells were seeded on the hBMP-6 coated surfaces, remarkable differences were observed. For cells seeded on layers of hBMP-6 on short (GPTMS) linkers, a much reduced, near negligible, amount of released hBMP- 6 protein was observed during the first 5 to 6 days. When cells were seeded on layers of hBMP- 6 on long (PGMA) linkers, the detected release profile was found close to the case without cells in the first 5 to 6 days. We also observed a reduced cell adhesion and spreading on PGMA layers in comparison with the GPTMS layers (Figure S2). A confluent cell layer was only reached after 7 days on the PGMA layers, while on GPTMS layers, confluency was reached at day 4. After 7 days, lower quantities of hBMP-6 were also detected in the medium on PGMA layers in the presence of cells. The observations indicate that when cells adhere earlier, as on GPTMS, less hBMP-6 escaped to the medium, and adhered cells with more bioactive hBMP-6 available, facilitates more interactions with cellular BMP receptors at the cell membrane. However, when cells adhere later, as on PGMA, slower released hBMP could still escape into the medium. These results confirm that release of hBMP-6 occurred and that the release kinetics could be decreased on brushes of PGMA, but for released hBMP- 6 to be confined to cells, as required for registration by cells, cells need to be adhered to the surface.

In a next experiment, KS483 cells were seeded for 4 days either in direct contact to or separated from the surface using a transwell assay (Figure 3 bottom). Low ALP activity was observed on control surfaces (glass, GPTMS and PGMA), irrespective of the presence of the transwell insert. Remarkably, when cells were seeded in direct contact to or in transwell above hBMP-6 immobilized on PGMA layers, in both of these cases, a lower ALP activity was observed in comparison when cells were seeded in direct contact to immobilized hBMP-6 on
GPTMS layers, while an intermediate ALP activity level was observed using GPTMS layers in the transwell assay. These observations confirm that cells seeded on GPTMS layers register more bioactive hBMP-6 after four days. This is presumably aided by adhered cells that are able to confine and register released hBMP-6 to produce ALP. This state of adhered cells and hence ability to confine released hBMP- 6 is different on PGMA layers, where both release of hBMP- 6 and cell adhesion is slower, but not to same extent slower.

The biological activity of released hBMP-6 was further confirmed by measuring the luciferase activity of $\mathrm{C} 2 \mathrm{C} 12$ cells that were stably transfected with a BMP-responsive element (BRE) fused to the firefly luciferase reporter gene (BRE-Luc cells) (Figure 4a). ${ }^{44} \mathrm{C} 2 \mathrm{C} 12$ is a model to study differentiation into bone cells. ${ }^{44}$ To avoid effects related to material stiffness, differences in luciferase expression relative to background of the same surface in absence of hBMP- 6 were used. Figure $4 a$ shows that luciferase activity was delayed to day two, when cells were seeded on surfaces with immobilized hBMP-6 using the short (GPTMS) linker in comparison to soluble hBMP-6, whereas on the long (PGMA) linker, a significant rise in luciferase activity was observed only at day seven. In addition, mRNA expression levels of osteoblast-specific runt-related transcription factor 2 (RUNX2) and osteocalcin $(\mathrm{OCN})$ genes were analyzed using quantitative PCR (Figure $4 \mathrm{~b}, \mathrm{c}$ ). ${ }^{45}$ Upregulation of RUNX2 was clearly observed already one day after cell seeding using either soluble hBMP-6 or hBMP-6 immobilized using the short linker. OCN expression was first observed after day three, which is in line with the delayed response of this gene during osteoblast differentiation. ${ }^{45,46}$ The increase in expression of the same genes when cells were seeded on hBMP-6 coated surfaces using the longer PGMA linker was clearly delayed. Taken together, these results confirm that the activation of ALP production and the induction of osteoblastic specific gene expression were activated by hBMP-6. Attachment of hBMP-6 to a surface 
a)

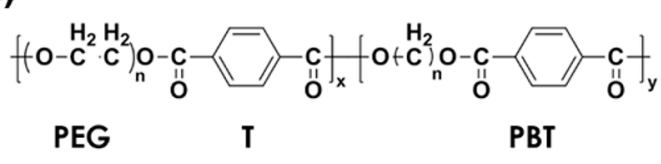

b)

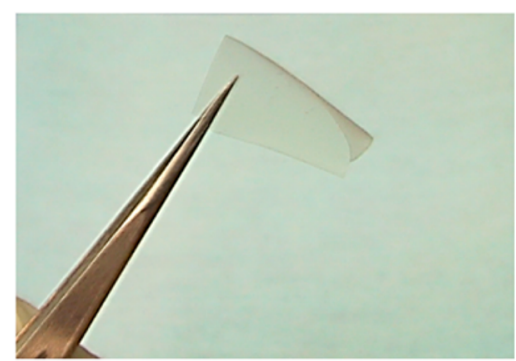

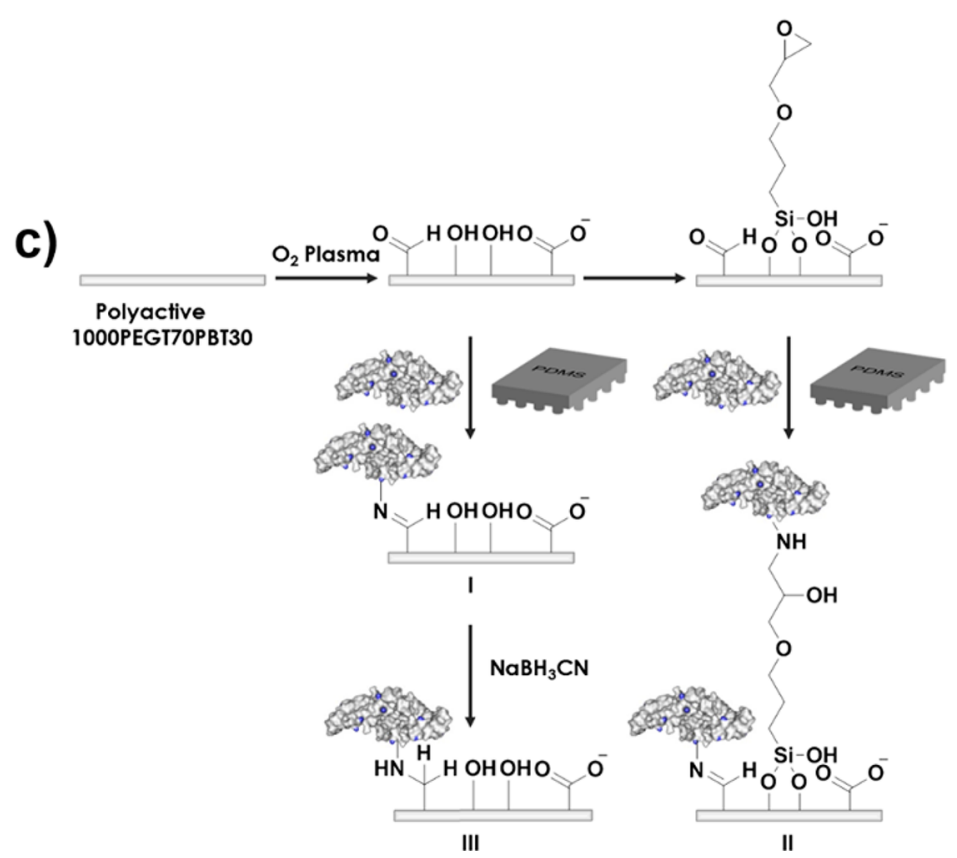

Figure 5. (a) Molecular structure of the block-copolymer (PA) consisting of a hydrophilic block of poly(ethylene glycol terephthalate) (PEGT) and a hydrophobic block of poly(butylene terephthalate) (PBT). (b) $100 \mu \mathrm{m}$ thick PA films were prepared by casting a solution of $3.4 \mathrm{wt} \% \mathrm{PA}$ in chloroform overnight and cured above $60{ }^{\circ} \mathrm{C}$ for several hours. (c) Schematic procedure of the surface functionalization of PA films after preactivation using $\mathrm{O}_{2}$ plasma, with GPTMS. hBMP-6 is subsequently immobilized via reactive $\mu \mathrm{CP}$ on the activated film via the reaction between the primary amine groups (accessible lysine residues in dark gray) and aldehyde groups forming an imine bond (I), which is further reduced in order to increase its stability toward hydrolysis (II) or on the GPTMS (III) with unreacted aldehyde groups also available to tether the growth factor as in I.

lessens its biological activity as compared to the soluble growth factor controls. The activity is unleashed by hydrolysis of the linker, which releases the hBMP-6 from the surface. These results are in agreement with the ELISA studies showing that in the case of the long linker, the release of hBMP-6 is delayed with 4 to 5 days before cells adhere and have a minimal dosage of released hBMP- 6 present that can activate ALP production and gene transcription. Therefore, we believe that the biological activity of hBMP-6 growth factors anchored via siloxane-based linkers is related to their release from the surface, but cells need to be adhered to confine the released growth factors to the cells.

Growth Factor Activity of hBMP-6 on Biomaterials. To demonstrate that our release strategy is applicable to polymeric biomaterials, we next attempted to functionalize a representative biomaterial with growth factors adopting our findings in line with our model study on glass. Therefore, a siloxane-bound GPTMS linker was attached on plasmaoxidized (Ox) $100 \mu \mathrm{m}$ thick films of commercially available biopolymers (Figure $5 \mathrm{a}$ and $5 \mathrm{~b}$ ) made of a poly(ether ester) copolymer (PA) comprising poly(ethylene oxide) (PEO) and poly(butylene terephthalate (PBT) onto which hBMP-6 was immobilized (Figure 5c, II). In a second set of experiments, hBMP-6 was immobilized directly onto this polymer surface after introduction of aldehyde groups by oxygen plasma treatment and the subsequent incubation with hBMP-6 to introduce imine bond formation (Figure 5c, I). A control surface with irreversibly bound GF was obtained by mildly reducing the imine bond (see the Supporting Information) to generate a stable, irreversible, secondary amine linkage under physiological conditions (Figure 5c, III). GPTMS-functionalization of PA was verified with contact angle measurements, XPS and FT-IR (Table S1, Figure S4). Upon oxidizing the PA surface the advancing contact angle decreased with respect to the native PA films, while upon coupling of GPTMS the contact angle increased. Upon reaction with GPTMS, the presence of elemental Si was detected in the XPS spectra, while deconvoluted XPS spectra of the C1s region (Figure S4) showed an increase of the peak at $286.35 \mathrm{eV}$, which can be attributed to ether bonds $(\mathrm{C}-\mathrm{O}) .{ }^{47}$ In addition, the peak centered at $288.52 \mathrm{eV}$, which can be mainly attributed to ester bonds $(\mathrm{O}-\mathrm{C}=\mathrm{O})$ that are present in native PA films, was 
broadened during plasma treatment indicating other carbonylcontaining species such as aldehyde/ketone $(\mathrm{C}=\mathrm{O})$ groups, which typically have bands in the range of $287.1-288.1 \mathrm{eV}$. In the FT-IR spectrum an $\mathrm{O}-\mathrm{H}$ stretching band appeared after plasma treatment of the native PA films (inset Figure S5a). This band was significantly reduced upon reaction with GPTMS indicating the conversion of $\mathrm{O}-\mathrm{H}$ bonds into O-Si bonds. Also, the ratio of the intensities of the asymmetric $\mathrm{C}-$ $\mathrm{O}-\mathrm{C}$ and the $\mathrm{C}=\mathrm{O}$ stretching bands (Figure $\mathrm{S} 5 \mathrm{~b}$ ) was inverted after the plasma treatment with a higher intensity for the carbonyl peak. Upon reaction with GPTMS, the ratio was inverted back with a slightly higher intensity for the $\mathrm{C}-\mathrm{O}-\mathrm{C}$ stretching band corresponding to the epoxide ring of the linker. Taken together, these results are indicative of successful activation of the PA films with groups that allow the immobilization of hBMP-6 as envisioned.

hBMP-6 microarrays on the activated PA films were prepared via reactive $\mu \mathrm{CP}$ as described in the model study on glass and visualized by immunostaining (Figure S6). The results showed that hBMP-6 was successfully anchored via the different immobilization strategies. For the GPTMS linker, a significant decay of fluorescence intensity was observed within two days, which is slower in comparison to the case of applying this linker on glass substrates, but faster than for immobilizing hBMP-6 via imines, while, after reduction using $\mathrm{NaBH}_{3} \mathrm{CN}$, the fluorescence intensity remained nearly constant over the course of seven days. This indicated that hBMP- 6 remained linked to the surface in this time period. Significant enhancement of cell adhesion was observed after the formation of a GPTMS linker on the PA films, irrespective of the presence of hBMP-6 (Figure S7d, h). Plasma treated PA films (PAOx) exhibited improved cell adhesion and spreading in comparison to native PA films (Figure S7a, b), which is in agreement with literature, ${ }^{48}$ while immobilization of hBMP-6 did not change cell adhesion as expected (Figure S7e, f). Similar cell adhesion behavior was found on these films after reduction of the imine bond (Figure S7g). The biological activity of the released hBMP-6 from PA films using the different linkers was verified using C2C12 BRE-Luc cells (Figure 6). The luciferase activity was increased when

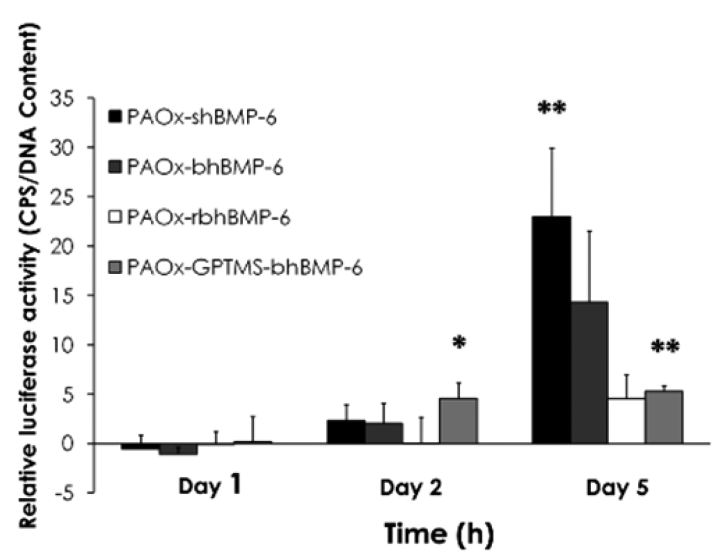

Figure 6. Luminescence of $\mathrm{C} 2 \mathrm{C} 12 \mathrm{BRE}-\mathrm{Luc}$ at different time points relative to the background. $*_{p}<0.05$ and $* *_{p}<0.01$. Surfaces: hBMP-6 supplemented to medium over PAOx (PAOx-shBMP-6), hBMP-6 bound to GPTMS on PAOx (PAOx-GPTMS-bhBMP-6), hBMP-6 bound as imine on PAOx (PAOx-bhBMP-6), and hBMP-6 bound via an amine resulting of the reduction of the imine (PAOxrbhBMP-6). Flat stamps were used for immobilization of hBMP-6. substantial quantities of hBMP-6 were released from the surface. For example, in the case of the GPTMS linker, a significant release of hBMP-6 after two days coincided with an increase in luciferase activity. Significant luciferase activity on imine films was only observed after five days, which indicates a slower release of hBMP-6. No luciferase activity was observed on surfaces where the imine bond was reduced into an irreversible secondary amine bond, which indicates effectively blocked release of hBMP-6 from the surface. These results confirm that the covalent attachment of hBMP-6 to a biomaterial surface was not biological active. This activity can subsequently be recovered after hydrolysis of the linker releasing the growth factor from the surface. By varying linker length (GPTMS versus PGMA using epoxide linkage) or linking strategy (imine versus secondary amine bonding), each with different susceptibility to hydrolysis and thus release from the growth factor from the surface, customizable bioactive surfaces can be engineered with spatio-temporal control over growth factor activation.

\section{CONCLUSIONS}

We have demonstrated the use of hydrolyzable linkers, such as siloxane, ester, and imine, for immobilization and regulated release of hBMP-6 to model surfaces as well as biomaterials. Immobilized hBMP-6 is biologically inactive. Upon release from the surface through hydrolysis of the covalent bonds its biological activity is recovered. We established a relation between the hydrolytic susceptibility of the bonds and osteoblastic cell differentiation. The use of labile strategies for the covalent attachment of growth factors to a surface with susceptibility for hydrolysis provides the means to engineer biomaterial surfaces with control over the dose of the growth factor to determine the release of bioactive growth factors over prolonged periods of time. We anticipate that this concept can be used to improve the design of a new generation of growth factor delivery systems in controlled drug delivery and for tissue engineering purposes.

\section{ASSOCIATED CONTENT}

\section{S Supporting Information}

The Supporting Information is available free of charge on the ACS Publications website at DOI: 10.1021/acs.langmuir.8b00853.

Materials and methods; AFM image and line profile of hBMP-6; effect of linker and immobilized hBMP-6 on cell adhesion and spreading; fluorescence microscopy images of immunostained micropatterned hBMP-6; XPS and FT-IRRAS spectra of PA films; advancing and receding water contact angles and selected XPS data of PA films (PDF)

\section{AUTHOR INFORMATION}

\section{Corresponding Authors}

*E-mail: p.jonkheijm@utwente.nl.

*E-mail: h.b.j.karperien@utwente.nl.

ORCID $\odot$

Jurriaan Huskens: 0000-0002-4596-9179

Pascal Jonkheijm: 0000-0001-6271-0049

Notes

The authors declare no competing financial interest. 


\section{ACKNOWLEDGMENTS}

Work by J.C-D. and E.L. was funded by Project P2.02 OAcontrol of the research program of the BioMedical Materials Institute, cofunded by the Dutch Ministry of Economic Affairs.

\section{REFERENCES}

(1) Hynes, R. O. The Extracellular Matrix: Not Just Pretty Fibrils. Science 2009, 326, 1216-1219.

(2) Tayalia, P.; Mooney, D. J. Controlled Growth Factor Delivery for Tissue Engineering. Adv. Mater. 2009, 21, 3269-3285.

(3) Yuasa, K.; Futamatsu, G.; Kawano, T.; Muroshita, M.; Kageyama, Y.; Taichi, H.; Ishikawa, H.; Nagahama, M.; Matsuda, Y.; Tsuji, A. Subtilisin-Like Proprotein Convertase Paired Basic Amino Acid-Cleaving Enzyme 4 Is Required For Chondrogenic Differentiation In ATDC5 Cells. FEBS J. 2012, 279, 3997-4009.

(4) Li, R. H.; Wozney, J. M. Delivering On The Promise Of Bone Morphogenetic Proteins. Trends Biotechnol. 2001, 19, 255-265.

(5) Ducy, P.; Karsenty, G. The Family Of Bone Morphogenetic Proteins. Kidney Int. 2000, 57, 2207-2214.

(6) Groeneveld, E.; Burger, E. Bone Morphogenetic Proteins In Human Bone Regeneration. Eur. J. Endocrinol. 2000, 142, 9-21.

(7) Ebisawa, T.; Tada, K.; Kitajima, I.; Tojo, K.; Sampath, T. K.; Kawabata, M.; Miyazono, K.; Imamura, T. Characterization Of Bone Morphogenetic Protein-6 Signaling Pathways In Osteoblast Differentiation. J. Cell Sci. 1999, 112, 3519-3527.

(8) Hudalla, G. A.; Murphy, W. L. Biomaterials That Regulate Growth Factor Activity Via Bioinspired Interactions. Adv. Funct. Mater. 2011, 21, 1754-1768.

(9) Lee, K.; Silva, E. A.; Mooney, D. J. Growth Factor DeliveryBased Tissue Engineering: General Approaches And A Review Of Recent Developments. J. R. Soc., Interface 2011, 8, 153-170.

(10) Cabanas-Danés, J.; Huskens, J.; Jonkheijm, P. Chemical Strategies For The Presentation And Delivery Of Growth Factors. J. Mater. Chem. B 2014, 2, 2381-2394.

(11) Crouzier, T.; Ren, K.; Nicolas, C.; Roy, C.; Picart, C. Layer-ByLayer Films As A Biomimetic Reservoir For rhBMP-2 Delivery: Controlled Differentiation Of Myoblasts To Osteoblasts. Small 2009, $5,598-608$

(12) Crouzier, T.; Fourel, L.; Boudou, T.; Albigès-Rizo, C.; Picart, C. Presentation Of BMP-2 From A Soft Biopolymeric Film Unveils Its Activity on Cell Adhesion and Migration. Adv. Mater. 2011, 23, H111-H118.

(13) Sakiyama-Elbert, S. E.; Hubbell, J. A. Controlled Release Of Nerve Growth Factor From A Heparin-Containing Fibrin-Based Cell Ingrowth Matrix. J. Controlled Release 2000, 69, 149-158.

(14) Herland, A.; Persson, K. M.; Lundin, V.; Fahlman, M.; Berggren, M.; Jager, E. W. H.; Teixeira, A. I. Electrochemical Control Of Growth Factor Presentation To Steer Neural Stem Cell Differentiation. Angew. Chem., Int. Ed. 2011, 50, 12529-12533.

(15) Chow, L. W.; Wang, L.-J.; Kaufman, D. B.; Stupp, S. I. SelfAssembling Nanostructures To Deliver Angiogenic Factors To Pancreatic Islets. Biomaterials 2010, 31, 6154-6164.

(16) Mammadov, R.; Mammadov, B.; Toksoz, S.; Aydin, B.; Yagci, R.; Tekinay, A. B.; Guler, M. O. Heparin Mimetic Peptide Nanofibers Promote Angiogenesis. Biomacromolecules 2011, 12, 3508-3519.

(17) Mammadov, R.; Mammadov, B.; Guler, M. O.; Tekinay, A. B. Growth Factor Binding on Heparin Mimetic Peptide Nanofibers. Biomacromolecules 2012, 13, 3311-3319.

(18) Mammadov, B.; Mammadov, R.; Guler, M. O.; Tekinay, A. B. Cooperative Effect Of Heparin Sulfate And Laminin Mimetic Peptide Nanofibers On The Promotion Of Neurite Outgrowth. Acta Biomater. 2012, 8, 2077-2086.

(19) Christman, K. L.; Vázquez-Dorbatt, V.; Schopf, E.; Kolodziej, C. M.; Li, R. C.; Broyer, R. M.; Chen, Y.; Maynard, H. D. Nanoscale Growth Factor Patterns by Immobilization on a Heparin-Mimicking Polymer. J. Am. Chem. Soc. 2008, 130, 16585-16591.
(20) Anderson, S. M.; Chen, T. T.; Iruela-Arispe, M. L.; Segura, T. The Phosphorylation Of Vascular Endothelial Growth Factor Receptor-2 (VEGFR-2) By Engineered Surfaces With Electrostatically Or Covalently Immobilized VEGF. Biomaterials 2009, 30, 46184628.

(21) Cabanas-Danés, J.; Dooms Rodrigues, E.; Landman, E.; van Weerd, J.; van Blitterswijk, C. A.; Verrips, T.; Huskens, J.; Karperien, M.; Jonkheijm, P. A Supramolecular Host-Guest Carrier System for Growth Factors Employing VHH Fragments. J. Am. Chem. Soc. 2014, $136,12675-12681$.

(22) Nakaji-Hirabayashi, T.; Kato, K.; Iwata, H. Essential Role Of Structural Integrity And Firm Attachment Of Surface-Anchored Epidermal Growth Factor In Adherent Culture Of Neural Stem Cells. Biomaterials 2008, 29, 4403-4408.

(23) Kuhl, P. R.; Griffith-Cima, L. G. Tethered Epidermal Growth Factor As A Paradigm For Growth Factor-Induced Stimulation From The Solid Phase. Nat. Med. 1996, 2, 1022-1027.

(24) Pompe, T.; Salchert, K.; Alberti, K.; Zandstra, P.; Werner, C. Immobilization Of Growth Factors On Solid Supports For The Modulation Of Stem Cell Fate. Nat. Protoc. 2010, 5, 1042-1050.

(25) Migliorini, E.; Valat, A.; Picart, C.; Cavalcanti-Adam, E. A. Tuning Cellular Responses To BMP-2 With Material Surfaces. Cytokine Growth Factor Rev. 2016, 27, 43-54.

(26) Corbett, P. T.; Leclaire, J.; Vial, L.; West, K. R.; Wietor, J.-L.; Sanders, J. K. M.; Otto, S. Dynamic Combinatorial Chemistry. Chem. Rev. 2006, 106, 3652-3711.

(27) Rowan, S. J.; Cantrill, S. J.; Cousins, G. R. L.; Sanders, J. K. M.; Stoddart, J. F. Dynamic Covalent Chemistry. Angew. Chem., Int. Ed. 2002, 41, 898-952.

(28) Moulin, E.; Cormos, G.; Giuseppone, N. Dynamic Combinatorial Chemistry As A Tool For The Design Of Functional Materials And Devices. Chem. Soc. Rev. 2012, 41, 1031-1049.

(29) Sreenivasachary, N.; Lehn, J.-M. Gelation-Driven Component Selection In The Generation Of Constitutional Dynamic Hydrogels Based On Guanine-Quartet Formation. Proc. Natl. Acad. Sci. U. S. A. 2005, 102, 5938-5943.

(30) Giuseppone, N.; Lehn, J.-M. Electric-Field Modulation of Component Exchange in Constitutional Dynamic Liquid Crystals. Angew. Chem., Int. Ed. 2006, 45, 4619-4624.

(31) Rozkiewicz, D. I.; Kraan, Y.; Werten, M. W. T.; de Wolf, F. A.; Subramaniam, V.; Ravoo, B. J.; Reinhoudt, D. N. Covalent Microcontact Printing Of Proteins For Cell Patterning. Chem. - Eur. J. 2006, 12, 6290-6297.

(32) Tauk, L.; Schröder, A. P.; Decher, G.; Giuseppone, N. Hierarchical Functional Gradients Of pH-Responsive Self-Assembled Monolayers Using Dynamic Covalent Chemistry On Surfaces. Nat. Chem. 2009, 1, 649-656.

(33) (a) Wasserman, S. R.; Tao, Y. T.; Whitesides, G. M. Structure And Reactivity Of Alkylsiloxane Monolayers Formed By Reaction Of Alkyltrichlorosilanes On Silicon Substrates. Langmuir 1989, 5, 10741087. (b) Tugulu, S.; Klok, H.-A. Stability and Nonfouling Properties of Poly(poly(ethylene glycol) methacrylate) Brushes under Cell Culture Conditions. Biomacromolecules 2008, 9, 906-912. (c) Deng, Y.; Zhu, X.-Y. A Nanotumbleweed: Breaking Away a Covalently Tethered Polymer Molecule by Noncovalent Interactions. J. Am. Chem. Soc. 2007, 129, 7557-7561. (d) Onclin, S.; Ravoo, B. J.; Reinhoudt, D. N. Engineering Silicon Oxide Surfaces Using SelfAssembled Monolayers. Angew. Chem., Int. Ed. 2005, 44, 6282-6304.

(34) Tsukruk, V. V.; Luzinov, I.; Julthongpiput, D. Sticky Molecular Surfaces: Epoxysilane Self-Assembled Monolayers. Langmuir 1999, 15, 3029-3032.

(35) Voskuhl, J.; Brinkmann, J.; Jonkheijm, P. Advances In Contact Printing Technologies Of Carbohydrate, Peptide And Protein Arrays. Curr. Opin. Chem. Biol. 2014, 18, 1-7.

(36) van der Horst, G.; van Bezooijen, R. L.; Deckers, M. M.; Hoogendam, J.; Visser, A.; Lwik, C. W.; Karperien, M. Differentiation Of Murine Preosteoblastic KS483 Cells Depends On Autocrine Bone Morphogenetic Protein Signaling During All Phases Of Osteoblast Formation. Bone 2002, 31, 661-669. 
(37) Wutzl, A.; Brozek, W.; Lernbass, I.; Rauner, M.; Hofbauer, G.; Schopper, C.; Watzinger, F.; Peterlik, M.; Pietschmann, P. Bone Morphogenetic Proteins 5 And 6 Stimulate Osteoclast Generation. J. Biomed. Mater. Res., Part A 2006, 77A, 75-83.

(38) Sui, X.; Di Luca, A.; Gunnewiek, M. K.; Kooij, E. S.; van Blitterswijk, C. A.; Moroni, L.; Hempenius, M. A.; Vancso, G. J. Stability And Cell Adhesion Properties Of Poly(N-isopropylacrylamide) Brushes With Variable Grafting Densities. Aust. J. Chem. 2011, 64, 1261-1268.

(39) Gao, H.; Elsabahy, M.; Giger, E. V.; Li, D.; Prud'homme, R. E.; Leroux, J.-C. Aminated Linear and Star-Shape Poly(glycerol methacrylate)s: Synthesis and Self-Assembling Properties. Biomacromolecules 2010, 11, 889-895.

(40) Gao, H.; Jones, M.-C.; Chen, J.; Prud'homme, R. E.; Leroux, J.C. Core Cross-Linked Reverse Micelles from Star-Shaped Polymers. Chem. Mater. 2008, 20, 3063-3067.

(41) Liang, Z.; Wu, X.; Yang, Y.-W.; Li, C.; Wu, G.; Gao, H. Quaternized Amino Poly(glycerol-methacrylate)s for Enhanced pDNA Delivery. Polym. Chem. 2013, 4, 3514-3523.

(42) Edmondson, S.; Huck, W. T. S. Controlled Growth And Subsequent Chemical Modification Of Poly(glycidyl methacrylate) Brushes On Silicon Wafers. J. Mater. Chem. 2004, 14, 730-734.

(43) Giannoudis, P.; Kanakaris, N.; Einhorn, T. Interaction Of Bone Morphogenetic Proteins With Cells Of The Osteoclast Lineage: Review Of The Existing Evidence. Osteoporosis Int. 2007, 18, 15651581.

(44) Logeart-Avramoglou, D.; Bourguignon, M.; Oudina, K.; Ten Dijke, P.; Petite, H. An Assay For The Determination Of Biologically Active Bone Morphogenetic Proteins Using Cells Transfected With An Inhibitor Of Differentiation Promoter-Luciferase Construct. Anal. Biochem. 2006, 349, 78-86.

(45) Ducy, P.; Schinke, T.; Karsenty, G. The Osteoblast: A Sophisticated Fibroblast Under Central Surveillance. Science 2000, 289, 1501-1504.

(46) Franceschi, R. T.; Xiao, G. Regulation Of The OsteoblastSpecific Transcription Factor, Runx2: Responsiveness To Multiple Signal Transduction Pathways. J. Cell. Biochem. 2003, 88, 446-454.

(47) Olde Riekerink, M. B.; Claase, M. B.; Engbers, G. H. M.; Grijpma, D. W.; Feijen, J. Gas Plasma Etching Of PEO/PBT Segmented Block Copolymer Films. J. Biomed. Mater. Res. 2003, 65A, 417-428.

(48) Claase, M. B.; Olde Riekerink, M. B.; de Bruijn, J. D.; Grijpma, D. W.; Engbers, G. H. M.; Feijen, J. Enhanced Bone Marrow Stromal Cell Adhesion And Growth On Segmented Poly(ether ester)s Based On Poly(ethylene oxide) And Poly(butylene terephthalate). Biomacromolecules 2003, 4, 57-63. 